\title{
Rituels langagiers des interactions enseignant / apprenants lors de Travaux Dirigés en Ecole d'Ingénieur
}

Quelles difficultés pour les étudiants allophones?

\section{Catherine Carras}

\section{OpenEdition}

\section{Journals}

Édition électronique

URL : http://journals.openedition.org/rdlc/817

DOI : $10.4000 /$ rdlc. 817

ISSN : 1958-5772

Éditeur

ACEDLE

Référence électronique

Catherine Carras, «Rituels langagiers des interactions enseignant / apprenants lors de Travaux

Dirigés en Ecole d'Ingénieur », Recherches en didactique des langues et des cultures [En ligne],

13-2 | 2016, mis en ligne le 25 septembre 2016, consulté le 21 avril 2019. URL : http:// journals.openedition.org/rdlc/817; DOI : 10.4000/rdlc.817

Ce document a été généré automatiquement le 21 avril 2019

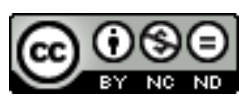

Recherches en didactique des langues et des cultures is licensed under a Creative Commons AttributionNonCommercial-NoDerivatives 4.0 International License 


\section{Rituels langagiers des interactions enseignant / apprenants lors de Travaux Dirigés en Ecole d'Ingénieur}

Quelles difficultés pour les étudiants allophones?

\section{Catherine Carras}

\section{Introduction}

Cette recherche s'inscrit dans le cadre du Français sur Objectif Universitaire (FOU) (Mangiante \& Parpette, 2011). Dans la perspective de la préparation linguistique des étudiants allophones se destinant aux filières scientifiques, nous nous intéressons spécifiquement aux étudiants souhaitant intégrer les écoles d'ingénieurs. En effet, on constate une présence massive du public allophone dans ces établissements : certaines écoles d'ingénieurs très tournées vers l'international, tel l'INSA de Lyon, accueillent près de $50 \%$ d'étudiants étrangers dans leurs différentes filières. Mettre en place des formations linguistiques, et par conséquent élaborer du matériel didactique à destination de ces publics, implique de se confronter à la réalité des discours auxquels ces étudiants doivent faire face dans leur domaine de spécialité, en collectant des données sur le terrain, dans la continuité de la démarche et des principes méthodologiques issus du Français sur Objectif Spécifique (FOS) (Mangiante \& Parpette, 2004).

Les étudiants allophones suivant des études supérieures dans une filière francophone se trouvent dans la situation d'acquérir en même temps des connaissances linguistiques et disciplinaires ; de même, ces connaissances disciplinaires leur sont transmises par le biais d'une langue qu'ils ne maîtrisent qu'en partie. Les forts taux d'échec constatés chez ces publics $^{1}$ sont en partie imputables à leur manque de préparation linguistique, surtout d'une préparation linguistique ciblée, prenant en compte la spécificité des discours, oraux et écrits, auxquels ils sont confrontés. 
3 Sans sous-estimer la part prépondérante de l'écrit dans les études supérieures, notamment lors des évaluations, il faut reconnaître que l'oral occupe une place fondamentale dans la transmission des connaissances. Les contenus disciplinaires sont certes accessibles aux étudiants par le biais des références bibliographiques (articles et ouvrages), des polycopiés de cours, etc., mais également, et nous croyons pouvoir affirmer en grande partie dans notre système universitaire, par le discours de l'enseignant pendant les cours.

4 Nous avons donc souhaité nous intéresser aux interactions orales en classe, aux usages langagiers que l'on peut y observer, et aux éventuelles implications didactiques que ces interactions peuvent induire, dans le contexte spécifique des études d'ingénieur effectuées par des étudiants allophones. Quels usages des différentes langues du répertoire des apprenants et des enseignantspeut-on observer dans les interactions pendant les cours en école d'ingénieur, notamment lors des travaux dirigés, où les échanges entre les divers acteurs sont intenses? Quelles difficultés de compréhension et d'appropriation ces interactions présentent-elles pour des étudiants allophones? Quelles en sont les conséquences didactiques pour les enseignants chargés de leur préparation linguistique?

\section{Cadre et objectifs de la recherche}

5 Dans le but de mieux cibler la formation linguistique des élèves ingénieurs allophones et d'élaborer des supports didactiques, nous avons été amenés à collecter diverses données auprès d'enseignants et étudiants de ces filières (supports de cours, polycopiés de cours, sujets d'examens, etc.) mais aussi de filmer des cours, Cours Magistraux (CM), Travaux Dirigés (TD) et Travaux Pratiques (TP). Cette collecte de données a été menée dans le cadre de l'élaboration d'un ouvrage didactique, manuel de FLE destiné aux élèves ingénieurs non francophones (Carras, Gewirtz \& Tolas, 2014).

6 Les $\mathrm{CM}$, mode de transmission très présent dans les universités françaises, leurs caractéristiques et les difficultés qu'ils peuvent représenter pour des étudiants allophones ont été étudiés par plusieurs auteurs : Bouchard et Parpette (2003 et 2007) en ce qui concerne leurs caractéristiques discursives, Parpette (2007) et Mangiante et Parpette (2011) pour les aspects discursifs et leur traitement didactique, Omer (2003) pour la prise de notes et ses difficultés pour les étudiants allophones, pour ne citer que ces quelques travaux. Les TD et TP, types de cours très présents dans les filières scientifiques, et donc dans les écoles d'ingénieur, n'ont quant à eux encore fait l'objet que de très peu de recherches dans le domaine du FOU. Il nous a semblé indispensable d'observer ces formats de cours, afin d'évaluer les compétences nécessaires à leur compréhension pour les étudiants allophones.

7 L'observation de ces différents types de cours a fait apparaître des formats interactionnels divers. Le $\mathrm{CM}$ est un discours monologal, dans la mesure où seul l'enseignant s'exprime, en continu, mais dialogique, car l'auditoire est pris en compte dans le discours de l'enseignant. Cette prise en compte du public apparaît à travers de nombreux marqueurs dialogiques qui constituent une gamme importante de procédés discursifs d'explicitation (répétitions, reformulations, exemplifications, etc.), mais aussi des traces du contexte d'énonciation (prise en compte du temps nécessaire à la prise de notes par les étudiants, par exemple), entre autres (Mangiante \& Parpette, 2011: 61). Ce 
dialogisme est « interlocutif » car le discours de l'enseignant est en relation directe avec les attitudes du public étudiant, qui manifeste soit silencieusement (cas le plus fréquent en $\mathrm{CM}$ ), soit verbalement ses réactions aux paroles de l'enseignant (ibid.). Le format monologal dialogique du CM devient polylogal dans le cas des TD et TP. L'observation d'un cours de TD fait ainsi apparaître une suite d'échanges verbaux, avec une alternance de tours de parole entre les différents acteurs, enseignant et étudiants. Dans les TD observés, la parole de l'enseignant occupe certes une place centrale, mais la parole des étudiants est également très présente. Ce type de cours se construit donc à travers des interactions. Le schéma interactif est encore un peu différent dans les TP, que nous pourrions qualifier de polylogal +, car il y a de fait le polylogue enseignant / étudiants, mais également de nombreuses interactions entre pairs, dans la mesure où les étudiants réalisent leurs manipulations en petits groupes, au sein desquels les interactions sont très fortes (et souvent gages de la réussite de la manipulation).

8 Ainsi, si dans un cours magistral, le format interactionnel exige rituellement que seul l'enseignant prenne la parole, il en va différemment lors des TD, et cette situation d'interaction peut être problématique pour les étudiants allophones d'un point de vue culturel et linguistique. D'un point de vue culturel, la situation d'interaction peut être source d'étonnement voire provoquer un choc didactique suivant la culture éducative d'origine des étudiants, en raison des positions interactionnelles des acteurs (la voix des étudiants a une place forte dans l'interaction, elle est très sollicitée par l'enseignant) : valeur accordée aux solutions proposées par les étudiants, possibilité de critique des propositions faites par l'enseignant de la part des étudiants, etc. Tout ceci peut venir bousculer les représentations des étudiants étrangers sur l'asymétrie statutaire entre enseignants et apprenants.

9 Tous ces aspects se traduisent en discours, et l'on voit apparaître des rituels langagiers qui structurent ce polylogue «de telle sorte que chaque participant sait quand il lui revient de prendre la parole et ce qu'il est en droit de dire» (Cicurel, $2011: 10$ ). Il faut donc être capable de décoder, d'un point de vue linguistique, les discours utilisés par les différents acteurs de l'interaction, afin d'en comprendre l'objet et de pouvoir y participer (même de façon passive). Les caractéristiques linguistiques de ces discours interactifs mettent fréquemment les étudiants allophones en difficulté pour plusieurs raisons, entre autres la rapidité de l'alternance des tours de parole, ou l'emploi d'un niveau de langue pouvant être considéré comme familier.

Ce sont donc ces caractéristiques des interactions que nous avons souhaité étudier dans le corpus observé. Ce dernierest constitué de captations vidéo de travaux dirigés de l'INP (Institut National Polytechnique) de Grenoble; il s'agit de TD d'Électrochimie, faisant suite à des $\mathrm{CM}$. Nous avons filmé environ 6 heures de ces cours. Nous avons observé et analysé les interactions enseignant / étudiants, en particulier lors de la correction collective des exercices. En effet, ces moments de correction collective concentrent les interactions enseignant / étudiants, et sont révélateurs, comme nous le verrons plus loin, du contrat didactique qui prévaut dans ce type de cours.

Dans la perspective de l'élaboration de matériel didactique visant la préparation linguistique des élèves ingénieurs allophones, les objectifs de cette recherche étaient les suivants :

- essayer de dégager des régularités (rituels) dans les interactions observées ;

- préciser le schéma interactionnel propre au type de cours observé (TD), indépendamment de la discipline ${ }^{2}$; 
- relever les modes privilégiés d'expression linguistique de ce schéma.

\section{Résultats}

\section{Rituels interactionnels}

12 rituels sont essentiels au bon fonctionnement des interactions dans des situations standardisées. Les rituels de politesse en sont le meilleur exemple : pratiques réglées, qui se reproduisent plus ou moins à l'identique dans des situations comparables, les rituels s'expriment au travers d'énoncés préformés syntaxiquement ou de simples moules susceptibles d'être remplis par du matériau linguistique très variable. En fonction des situations de communication, ces rituels vont avoir plus ou moins d'importance. Une consultation médicale est ainsi beaucoup plus ritualisée qu'une conversation familière. Lorsque le cadre de l'échange est défini institutionnellement, le comportement langagier des différents acteurs est soumis à certaines restrictions (Lüger, 1993: 6), et le respect des rituels conditionne en partie la réussite de l'échange.

Cest ce qui se passe dans une salle de classe : le cadre est prédéfini, le rôle des différents acteurs également. Dans la communication didactique, l'enseignant met en discours son savoir afin de le faire partager à son public (Ali Bouacha, 1984). Cicurel (2011: 10) a souligné le caractère ritualisé des échanges en classe : ces échanges verbaux ont la particularité de mettre en présence des acteurs au statut asymétrique, statut défini par l'institution. L'enseignant dirige les échanges, les apprenants participent au polylogue et influencent sa dynamique.

Les travaux de Cicurel portent sur la classe de langue, mais nombre de ses remarques s'appliquent aux TD que nous avons observés, même si l'objet d'enseignement n'est pas la langue mais une discipline scientifique. Tout d'abord, parce que ce sont les interactions qui ont retenu notre attention; ensuite, parce que nous avons observé et analysé cette situation didactique avec un regard d'enseignant de langue, dans une perspective de formation linguistique.

15 Nous avons tenté de dégager des régularités dans les échanges enseignant / étudiants, régularités qui permettraient d'isoler certains éléments prévisibles, afin de préparer le public d'étudiants allophones avec lequel nous travaillons à mieux s'intégrer dans ce type d'échanges et à savoir ce que l'on attend de lui en tant qu'acteur.

16 L'observation des moments de correction collective des exercices a fait apparaitre deux rituels auxquels nous allons nous attacher :

- la co-construction de la solution : l'enseignant ne «donne » pas la solution aux étudiants, mais les amène progressivement vers une résolution collective du problème ;

- la négociation: chaque participant à l'échange, enseignant et étudiant, argumente en faveur d'une solution.

Ce type d'échange, qui apparaît de façon récurrente dans les cours que nous avons observés, montre qu'à l'intérieur d'une situation en partie ritualisée, le discours est tout de même co-construit et co-géré par les différents acteurs. En cela, ces échanges constituent des indices du contrat didactique qui régit ces cours. Les différents acteurs acceptent qu'il y ait des règles pour qu'il y ait apprentissage, et le contrat qui prévaut ici est que chacun doit participer à la construction de la solution : les étudiants acceptent

Recherches en didactique des langues et des cultures, 13-2 | 2016 
que l'enseignant ne leur délivre pas la solution dès le départ, et l'enseignant accepte que les étudiants puissent avoir des propositions différentes de la sienne.

Dans les exemples ci-dessous, il apparaît assez clairement que l'enseignant énonce des règles de participation, et explicite le contrat didactique :

$\mathrm{P}^{3}$ : Donc je vous laisse un peu réfléchir parce que volontairement y'a ... la solution n'est pas forcément unique voilà !

$\mathrm{P}$ : Ouais c'est une idée ... donc on peut prendre effectivement une valeur médiane. Est-ce qu'il y a pas quelque chose d'autre ou une autre méthode, est-ce qu'il y a pas quelqu'un qui a une autre idée ? Enfin on est là pour discuter hein!

$P$ : Est-ce que quelqu'un a une idée ? C'est extrêmement simple donc si je vous donne la solution j'en ai pour 30 secondes, je vous amène à réfléchir.

La co-construction de la solution apparaît ici comme faisant partie intégrante du contrat didactique, et l'enseignant ressent le besoin d'expliciter ce contrat : l'apprentissage ne se fera pas si la solution est donnée par l'enseignant, le contrat consiste à réfléchir par soimême; de plus, il n'y a pas forcément une solution unique, ce qui implique des échanges de négociation.

Voici deux échanges où ce rituel apparaît clairement. Premier échange :

E : Moi j'ai un truc ! Comme on connait la puissance, on connaît la densité, on a la surface aussi des cellules, donc on sait que $U$ fois la densité de courant doit être égal à 5000 watts sur les $300 \mathrm{~m} 2$.

P : D'accord ...

$\mathrm{E}$ : Donc en fait il faut trouver un couple tension / densité qui fasse 16,6.

$\mathrm{P}:$ Qui fasse?

$\mathrm{E}: 16,6$.

$\mathrm{P}: 16,6$ quoi ?

E : Ben c'est des volts !

$\mathrm{P}$ : Pourquoi cette valeur que vous m'annoncez?

E : En fait c'est 5000 sur $300 \ldots$

P : Ah excusez-moi j'avais pas compris d'accord! OK mais 5000 sur 300 pourquoi 300 ? Ouais mais là 5000 sur 300 j'ai un peu de mal à voir à quoi ça va servir de diviser une puissance par une surface!

$\mathrm{E}$ : Le courant c'est égal à la surface fois la densité de courant ...

$\mathrm{P}:$ Le courant c'est ... oui tout à fait (...)

21 construction du savoir (voir les règles définies plus haut par l'enseignant). On voit ici un étudiant qui propose sa propre solution ("Moi j'ai un truc!»), l'enseignant qui accueille sa proposition et l'encourage à poursuivre (" D'accord ...» $\left.{ }^{4}\right)$. Vient ensuite une alternance de tours de paroles où l'on remarque des réajustements et demandes / apports de précisions quant à la proposition.

Deuxième échange :

$\mathrm{P}$ : Mais y'en aura pas qu'un, à mon avis il y en aura plus d'un alors est-ce qu'on est d'accord que là, on peut prendre n'importe quel point, on $y$ arrivera ? Comment on pourrait raisonner intelligemment?

$\mathrm{E}$ : Mais non on pourra pas prendre n'importe quel point !

$\mathrm{P}$ : Pourquoi?

$\mathrm{E}:$ Il faut un point qui est sur la courbe quand même !

$\mathrm{P}$ : Ah ben oui! Oui bien sûr, on peut prendre n'importe quel point de la 
courbe. Je peux me mettre à 1 ampère, à 1,5 à 0,5 à 0,1 j'arriverai toujours ...

$\mathrm{E}: \mathrm{J}$ 'arriverai pas à respecter le rapport !

$\mathrm{P}:$ Ah si vous y arriverez toujours ! est d'accord ... $\left.»^{5}\right)$. On voit également que l'étudiant peut contester la solution proposée par l'enseignant, il peut même l'interrompre (voir la fin de l'échange) et contester sa proposition. Dans cette classe de TD, en tant que lieu social ayant ses propres règles de communication, on peut donc observer que les positions interactionnelles se manifestent discursivement (Cicurel, 2011 :11). Il s'agit d'une situation didactique, le statut des différents acteurs est donc asymétrique, en particulier, l'enseignant aura toujours «le dernier mot ", mais on constate que la position des étudiants peut être considérée comme haute, leur voix est non seulement sollicitée mais valorisée, et en ce sens cette situation constitue un véritable polylogue.

\section{Routines interactionnelles}

Nous avons souhaité faire une distinction entre rituels et routines, dans la mesure où les éléments auxquels nous allons nous intéresser ici sont des éléments verbaux, constituants d'actes de langage. Cette distinction a été établie par Traverso (1996: 41): «rituel» désigne globalement le type de comportement et «routine» renvoie à la réalisation particulière du rituel. En d'autres termes, "saluer à l'ouverture d'une interaction est un comportement rituel; le faire en disant " bonjour ", "salut », " ciao", " hello", "quelle bonne surprise » sont autant de routines » (ibid.).

Par « routine ", nous entendons des activités langagières récurrentes qui s'appuient sur des formulations plus ou moins standardisées. Les routines que nous avons dégagées de notre corpus d'observation ne constituent pas des formulations standardisées à proprement parler, telles que les exemples de salutation évoqués ci-dessus, mais la réitération de certaines caractéristiques linguistiques dans les échanges nous ont semblé dignes d'intérêt, compte tenu du public auquel nous nous adressons.

Toujours selon Traverso (1996), c'est justement grâce à leur caractère préfabriqué que les routines facilitent les interactions. Lorsqu'elles sont très standardisées, ces routines sont en partie «vides de sens » pour les membres du groupe dans lequel elles sont en usage. Or, pour l'apprenant d'une langue étrangère, qui ignore en grande partie ces routines, elles ne sont absolument pas vides de sens, et leur maîtrise insuffisante peut provoquer des ruptures dans l'interaction.

Nous pensons qu'il en est souvent ainsi dans les réalisations langagières des rituels décrits ci-dessus. Dans les exemples que nous allons présenter ci-après, les formulations en partie préfabriquées, en tout cas relativement prévisibles pour des locuteurs natifs, peuvent avoir un sens difficilement accessible pour des non-natifs.

Nous souhaiterions observer quelques exemples qui, selon nous, peuvent se révéler particulièrement problématiques pour des étudiants allophones en raison des spécificités suivantes :

- la rapidité des tours de parole

- l'emploi d'un registre de langue pouvant être considéré comme familier

- la présence d'énoncés peu explicites quant à l'action à réaliser

- la présence d'énoncés à double signification 
pas possible d'illustrer la rapidité des tours de parole par des exemples cités, mais le fait qu'il s'agit d'un polylogue, où, comme nous l'avons vu, la voix des différents acteurs a un poids sinon équivalent du moins comparable, fait que les échanges s'effectuent à un rythme soutenu. Cette rapidité peut effectivement poser des problèmes aux étudiants allophones, qui auront du mal à accompagner ces interactions, et encore plus à y prendre part.

De nombreux exemples d'emploi d'un registre de langue pouvant être considéré comme familier, tant de la part des étudiants que de l'enseignant, ont été relevés :

E : Moi j'ai un truc ! (...)

$\mathrm{P}$ : Là au début ça sert à rien, on s'en fout, on va tracer à la louche.

$\mathrm{E}$ : Ouais, c'est la méthode empirique comme on dit ...

$\mathrm{P}:$ Ah ben oui de toute manière !

On comprend aisément que ces échanges soient déroutants pour des étudiants allophones, tout d'abord d'un point de vue linguistique (l'expression «tracer à la louche » ne fait pas forcément partie de leur répertoire), mais également pour des questions culturelles. Dans certaines cultures, il n'est probablement pas envisageable qu'un étudiant prenne la parole pour proposer une solution en commençant par «moi j'ai un truc!", ni que l'enseignant affirme que la précision n'est pas importante dans cette phase de l'exercice en disant « on s'en fout ».

On relève également des exemples d'énoncés peu explicites quant à l'action attendue :

$P$ : On vous a pas beaucoup entendus là-bas!

$\mathrm{P}$ : Est-ce qu'il y a pas quelqu'un qui a une autre idée?

$\mathrm{P}$ : Est-ce qu'il y a pas quelque chose d'autre?

Ces énoncés peu explicites peuvent être considérés comme des actes illocutoires indirects (Traverso, 1996 : 41). Ainsi, de la même façon qu'un énoncé tel que « avez-vous l'heure? » doit être interprété comme "quelle heure est-il?», le premier exemple doit être interprété non pas véritablement comme un reproche (ce que pourraient faire des allophones), mais comme une invitation (ou plutôt une injonction) à participer. Dans les deux derniers exemples, l'enseignant signale implicitement que la solution proposée précédemment n'est pas la bonne (tout en ne disant pas «non ce n'est pas cela» ou « c'est faux »), et qu'il faut continuer à chercher.

De même, on remarque l'emploi marqué de mots qui peuvent assumer des significations contradictoires en fonction du contexte et de l'intonation. Tout comme le mot «merci» peut signifier que l'on accepte ou que l'on refuse une proposition, l'enseignant utilise régulièrement les mots " ouais " et "d'accord" pour exprimer autre chose que son accord, l'intonation montrant qu'il invite, en fait, les étudiants à poursuivre leur intervention. Remarquons d'ailleurs ici une différence fondamentale entre "oui » et « ouais » en ce qui concerne l'assertion, nuance qui peut échapper à des allophones. On se situe bien ici dans le cadre des routines en grande partie standardisées. 


\section{Quelles difficultés pour les étudiants allophones?}

\section{Schéma interactionnel}

Les exemples cités, et de manière générale l'analyse des échanges dans les cours observés, montrent un schéma interactionnel problématique pour les étudiants allophones aussi bien au niveau des rituels, en particulier en ce qui concerne la position interactionnelle des différents acteurs, que des routines, susceptibles de poser des problèmes de décodage linguistique.

La position interactionnelle des différents acteurs peut, suivant les cultures d'origine des étudiants étrangers, provoquer un choc didactique ou culturel. En effet, il s'agit d'une situation didactique, et il existe effectivement une asymétrie statutaire entre les différents acteurs. Toutefois, comme nous l'avons vu dans les exemples cités, cette asymétrie se marque surtout par le fait que l'enseignant est l'animateur des échanges, du polylogue, il n'est pas le seul acteur dans la construction du savoir, même si, in fine, c'est bien lui qui valide les solutions proposées par les étudiants. Le contrat didactique dans ce type de cours (qui est en partie explicité par l'enseignant, comme nous l'avons vu plus haut), stipule queles étudiants peuvent et doivent intervenir, et que leur voix a une place forte, très sollicitée par l'enseignant. Cette position interactionnelle haute des étudiants dans un TD peut constituer une difficulté pour des étudiants étrangers. Tout d'abord pour des raisons d'insécurité linguistique : il faut en effet oser prendre la parole spontanément devant tout le groupe classe, sans être sûr que la solution proposée sera validée. Ensuite, compte tenu du statut des différents acteurs face au savoir: dans certaines cultures scolaires ou universitaires, l'enseignant est le seul détenteur du savoir, et l'on n'intervient pas si l'on n'est pas sûr de ce que l'on va dire, alors qu'au contraire dans les cours observés l'erreur est acceptée. On comprend bien que pour certains étudiants, en particulier asiatiques, cette situation puisse provoquer un véritable choc didactique.

Ainsi, si l'on considère spécifiquement les publics chinois, il semble bien que le cours de TD tel que nous le connaissons dans nos filières ne soit pas la norme, comme l'illustre le témoignage d'une enseignante de physique-chimie française intervenant dans une école d'ingénieur filière francophone en Chine :

Les TD en petits groupes où ce sont les étudiants qui doivent faire les exos et le prof est seulement là pour animer la séance, corriger les erreurs si les étudiants n'y arrivent pas et éventuellement indiquer des méthodes différentes ou faire des remarques [n'existent pas ici]. D'après les échanges que j'ai eus avec mes collègues de maths à ce sujet, ce n'est pas le cas en Chine: ni en terme de petits groupes, ni en terme de participation. En général c'est le professeur qui corrige des exos en classe entière sans participation. (Ding, $2015: 55$ )

Si l'on s'attache plus particulièrement aux éventuelles difficultés linguistiques de ces publics face à ce type d'échange, on constate tout d'abord, répétons-le, que suivre un cursus en français implique pour eux un apprentissage simultané des contenus disciplinaires et de la langue. Mais aussi, comme nous l'avons vu dans les différents exemples cités, la place centrale des interactions verbales dans ce type de cours (interactions enseignants / étudiants mais également entre pairs, lors des moments de réalisation d'exercices) et leurs caractéristiques linguistiques et discursives, engendrent une difficulté très importante, voire insurmontable pour certains étudiants, quand il s'agit d'être véritablement acteurs dans ces interactions. Car cela suppose, tout d'abord, 
de décoder les échanges (qu'attend de moi l'enseignant lorsqu'il dit "On vous a pas beaucoup entendus là-bas!»), mais aussi d'être assez sûr de son niveau de langue pour prendre la parole face au groupe. Intervient ici une notion qui nous semble fondamentale, qui est celle de la face des différents acteurs dans ces interactions. En effet, intervenir sans être sûr de sa solution, en utilisant un registre de langue éventuellement familier, contester la solution proposée par l'enseignant, ne met en danger la face d'aucun des acteurs, ce qui est loin d'être la norme dans toutes les cultures universitaires.

\section{Le discours scientifique : un discours construit}

La co-construction du savoir observée dans ces échanges tient non seulement au contrat didactique, mais constitue également une trace de la spécificité du discours scientifique en tant que discours construit. En effet, le discours didactique scientifique est, dans la plupart des cas, construit à partir de la démarche scientifique :

Une observation conduit à la formulation d'un problème scientifique presque toujours sous forme d'une question; pour trouver une solution à ce problème, des hypothèses sont émises; ces hypothèses seront ou non validées par la mise en place d'expériences et d'observations. (...) La validation de l'hypothèse se conclut par la formulation d'un savoir scientifique nouveau (Gapaillard \& Feutry Le Pennec, 2006 : 12).

Cette construction du savoir, lors des TD et TP, est collective, les étudiants énoncent euxmêmes des hypothèses, les vérifient ou non lors des expérimentations, afin de formuler de façon collective un savoir nouveau. C'est cette démarche que l'enseignant initie lorsqu'il dit «si je vous donne la solution j'en ai pour 30 secondes, [alors] je vous amène à réfléchir ». Les interactions en classe reflètent et participent à cette co-construction. Les difficultés plus ou moins grandes des étudiants étrangers à être acteurs dans ces interactions de co-construction du savoir risquent de les placer en-dehors de ce qui est attendu d'un apprenti scientifique. On voit donc que les difficultés potentielles de ces publics dépassent le cadre linguistique et méthodologique, et tiennent aussi au positionnement face au discours scientifique.

\section{Pistes didactiques}

La préparation linguistique des étudiants allophones à intégrer nos filières universitaires passe nécessairement par la confrontation avec la réalité des discours, didactiques ou autres, qui circulent dans ces milieux. D'où l'importance d'une collecte de données sur le futur terrain d'exercice de ces publics. Les captations vidéos analysées dans cet article ont été exploitées dans l'ouvrage cité plus haut (Carras et al., 2014). Dans les deux fiches pédagogiques consacrées à la compréhension des Travaux Dirigés, les caractéristiques liées aux interactions enseignants / étudiants font l'objet d'activités, même si elles ne sont pas forcément centrales. Dans un tel ouvrage, au nombre de pages limitées, et dans lequel on s'efforce de couvrir l'ensemble des compétences visées, s'attarder sur un point précis est relativement difficile. Néanmoins, le support vidéo figurant dans le manuel permet aux enseignants qui le souhaiteraient d'exploiter plus finement certains passages. Nous pouvons toutefois donner quelques exemples d'exploitation. différentes fonctions des énoncés peu explicites employés par l'enseignant ou les 
étudiants, ainsi que le montre l'exemple qui suit (Carras et al., 2014: 75-76) (Tableaux 1 et 2) :

Consigne : Regardez la vidéo N 13 «Échanges enseignant / étudiants ».

Voici des mots ou expressions utilisés par l'enseignant pour réagir aux propos des étudiants. À votre avis, quelles sont leurs fonctions dans le discours de l'enseignant? Attention, le ton utilisé par l'enseignant et le contexte sont essentiels !

Tableau 1

\begin{tabular}{|c|c|c|c|c|c|}
\hline & $\begin{array}{l}\text { Bonne } \\
\text { suggestion }\end{array}$ & $\begin{array}{l}\text { Mauvaise } \\
\text { suggestion }\end{array}$ & $\begin{array}{l}\text { Les deux, en } \\
\text { fonction du } \\
\text { contexte }\end{array}$ & $\begin{array}{l}\text { Encourage à } \\
\text { continuer }\end{array}$ & $\begin{array}{l}\text { Demande une } \\
\text { précision / une } \\
\text { explication }\end{array}$ \\
\hline Ouais & & & & & \\
\hline D'accord & & & & & \\
\hline $\begin{array}{l}\text { Je suis d'accord } \\
\text { avec vous }\end{array}$ & & & & & \\
\hline Pourquoi? & & & & & \\
\hline $\begin{array}{l}\text { Ouais, c'est une } \\
\text { idée }\end{array}$ & & & & & \\
\hline $\begin{array}{l}\text { Est-ce qu'il y a } \\
\text { pas quelque } \\
\text { chose d'autre? }\end{array}$ & & & & & \\
\hline $\begin{array}{l}\text { Est-ce qu'il y a } \\
\text { pas quelqu'un } \\
\text { qui a une autre } \\
\text { idée? }\end{array}$ & & & & & \\
\hline $\begin{array}{l}\text { J'ai un peu du } \\
\text { mal à voir à quoi } \\
\text { ça va servir }\end{array}$ & & & & & \\
\hline Tout à fait & & & & & \\
\hline $\begin{array}{l}\text { On vous a pas } \\
\text { beaucoup } \\
\text { entendus là-bas! }\end{array}$ & & & & & \\
\hline
\end{tabular}

Et maintenant, voici des mots ou expressions utilisés par les étudiants, classez-les également : 


\begin{tabular}{|c|c|c|c|c|}
\hline & $\begin{array}{l}\text { Pour faire } \\
\text { une } \\
\text { proposition }\end{array}$ & $\begin{array}{l}\text { Pour } \\
\text { expliquer } \\
\text { ma solution }\end{array}$ & $\begin{array}{lr}\text { Pour dire } \\
\text { que je ne } \\
\text { suis pas } \\
\text { d'accord }\end{array}$ & $\begin{array}{ll}\text { Pour dire que } \\
\text { je ne } \\
\text { comprends } \\
\text { pas }\end{array}$ \\
\hline Moi j'ai un truc! & & & & \\
\hline $\begin{array}{l}\text { C'est quoi ce que vous avez } \\
\text { noté? }\end{array}$ & & & & \\
\hline $\begin{array}{l}\text { On a la surface de chaque } \\
\text { cellule et on en déduit la densité } \\
\text { de courant. }\end{array}$ & & & & \\
\hline Donc, en fait, il faut trouver (...) & & & & \\
\hline $\begin{array}{l}\text { Mais non, on pourra pas } \\
\text { prendre n'importe quel point! }\end{array}$ & & & & \\
\hline $\begin{array}{l}\text { Et là, c'est ce qu'on a U fois la } \\
\text { densité, donc je cherche à } \\
\text { trouver un couple qui vaut ce } \\
\text { rapport. }\end{array}$ & & & & \\
\hline $\begin{array}{l}\text { Il faut un point qui est sur la } \\
\text { courbe quand même! }\end{array}$ & & & & \\
\hline $\begin{array}{l}\text { Comme on connaît la puissance } \\
\text { on a I, on a la surface aussi des } \\
\text { cellules, donc on sait que U fois } \\
\text { la densité de courant doit être } \\
\text { égal à } 5000 \text { watts sur les } 300 \mathrm{~cm}^{2}\end{array}$ & & & & \\
\hline
\end{tabular}

Les expressions exprimant l'imprécision ont été travaillées à partir de transcriptions. Le choix des auteurs s'est porté sur cette modalité de travail dans l'objectif de faire découvrir et repérer cette caractéristique des discours observés et leur expression linguistique avant de confronter les apprenants aux discours oraux.

Exemple d'exercice (Carras et al., 2014 : 76):

Consigne: Dans la transcription ci-dessous, retrouvez les expressions, utilisées par l'enseignant ou les étudiants, qui indiquent l'imprécision :

Enseignant : Alors, essayez de me trouver le résultat. Là, vous tracez dessus, à la louche hein! il s'agit pas de faire du point par point! vous prenez la valeur du courant et puis, vous essayez de retrouver la position de la valeur de puissance pour voir dans quelle gamme on est, hein ! c'est environ ... il s'agit pas de trouver exactement le point au milliampère près, c'est à la cinquantaine de milliampère, il suffit de savoir si on est plutôt proche de 1 ampère au $\mathrm{cm}^{2}$ ou plutôt 800 ou plutôt 750 comme certains l'ont fait.

Étudiant : il faut calculer tous les points? 
Enseignant : vous les prenez tous, oui, c'est rapide ! Là, au début, ça sert à rien, on s'en fout, on va tracer à la louche, c'est plutôt dans ces zones-là que ça va être intéressant, donc c'est sûr que ça sera pas forcément par là qu'il sera le maximum. Vous rajoutez un axe sur les bords et puis ... vous devez pas être très très loin, vous, je pense avec votre valeur. Ouais ouais, c'est environ, mais prenez un axe des puissances plutôt que de marquer dessus !

Étudiant : ouais, c'est la méthode empirique, comme on dit ...

Enseignant : ah, ben oui, de toute manière !

Les expressions appartenant au registre familier font l'objet de la même modalité de travail.

\section{Exemple d'exercice (Carras et al., 2014 : 70) :}

Consigne : L'enseignant utilise dans son discours des expressions familières, les comprenez-vous?

On va attaquer les séances de TD.

Les deux [thèmes] seront à l'examen, faut pas croire!

Nos téléphones portables, nos ordinateurs, ils sont toujours en rade à cause des batteries!

Les atomes comment c'est foutu?

Ces quelques exemples d'exploitation ne constituent bien entendu que des pistes. Notre intention est de montrer la nécessité de la prise en compte des caractéristiques des discours didactiques dans leur ensemble, sans se limiter aux aspects purement terminologiques (qui sont, par expérience, rarement ceux qui posent le plus de problèmes de compréhension aux publics allophones).

Pour les questions interculturelles, en particulier celle d'un éventuel choc didactique par rapport à des pratiques très différentes dans les pays d'origine des étudiants, des moments d'échange peuvent être organisés pendant la classe de langue. Ces contrastes sont difficiles à prévoir car ils dépendent en grande partie de la culture et des expériences antérieures des publics. Néanmoins, de nombreuses études ont été consacrées au caractère interculturel de l'intégration universitaire. Pour ce qui concerne les publics d'élèves ingénieurs allophones, citons: Samper Mangin (2011) qui s'attache à l'hétérogénéité des publics multinationaux en école d'ingénieur ; Ding (2015) dont l'étude porte sur les étudiants chinois suivant des études en filières d'ingénieur francophone; enfin, Toshimitsu Fujita (2011) qui traite particulièrement de l'interculturalité dans les formations d'intégration des élèves ingénieurs brésiliens. Ces différentes études montrent que le choc didactique est une réalité. C'est à l'enseignant d'en prendre conscience et de mettre en place des activités d'échange, éventuellement en langue maternelle si le contexte et la composition du public le permettent, afin de préparer au mieux les étudiants allophones à ces pratiques différentes.

\section{Conclusion}

Les observations menées viennent confirmer ce qui a déjà été dit à propos du FOU : une véritable prise en compte des besoins des étudiants allophones passe par une analyse des discours universitaires auxquels ils sont véritablement confrontés dans les différentes filières. La place fondamentale des interactions de co-construction du savoir dans les TD, auxquelles il est indispensable de préparer les nombreux étudiants étrangers intégrant des écoles d'ingénieur, ne nous est apparue que grâce aux observations directes de cours. 
Cela peut sembler une évidence, mais en tant qu'enseignant de langue, la représentation que l'on peut avoir des types de discours qui circulent dans les filières scientifiques est parfois loin de la réalité, et seule une collecte de données sur le terrain permet d'évaluer précisément les besoins de formation linguistique et méthodologique des étudiants allophones.

Les CM occupent certes une place importante dans la transmission des connaissances en école d'ingénieur, mais une sensibilisation aux caractéristiques interactionnelles et leurs réalisations linguistiques en TD et TP doit nécessairement figurer dans un programme de préparation linguistique pour élèves ingénieurs allophones. Ainsi, il convient d'accorder une place importante à la compétence interactionnelle, et de ne pas limiter la formation linguistique de ces publics aux compétences de réception et production, orale et écrite. Comme l'observation de cours le montre, cette compétence interactionnelle est indispensable non seulement dans le processus d'appropriation langagière, mais également d'acquisition de connaissances disciplinaires.

Dans la continuité de ces recherches, nous souhaiterions à présent nous pencher plus spécifiquement sur les interactions entre pairs, notamment lors de la réalisation d'expérimentations en petits groupes lors des TP. En effet, ces interactions participent à la démarche scientifique telle que nous l'avons évoquée plus haut. La capacité àêtre acteur dans cette situation didactique est une compétence indispensable pour le public d'élèves ingénieurs allophones auquel nous nous intéressons. Il convient donc d'intégrer dans les programmes de formation en FOU, et par conséquent dans la formation des enseignants chargés de ces programmes, la nécessaire prise en compte de ces interactions, qui sont souvent éloignées de la représentation habituelle des «discours spécialisés». Le discours scientifique didactique ne se construit pas seulement par le biais d'une terminologie spécifique, mais également, en tant que discours construit, par les interactions en situation de cours.

\section{BIBLIOGRAPHIE}

Ali Bouacha, A. (1984). Le discours universitaire: la rhétorique et ses pouvoirs. Berne-Francfort : Peter Lang.

Bouchard, R. \& Parpette, C. (2007). « Gestion lexicale et prise de notes dans les cours magistraux : le cas des CM de droit ».LIDIL, n³5. Grenoble : Ellug. pp. 199-209.

Carras, C., Gewirtz, O. \& Tolas, J. (2014). Réussir ses études d'ingénieur en français. Grenoble : PUG.

Charaudeau, P. \& Maingueneau, D. (2002). Dictionnaire d'analyse du discours. Paris : Seuil.

Cicurel, F. (2011). Les interactions dans l'enseignement des langues. Agir professoral et pratiques de classe. Paris : Didier. 
Ding, X. B. (2015). Conception et implémentation d'un programme de Français sur objectif universitaire en école d'ingénieur implantée en Chine. Mémoire de Master 2 Sciences du Langage / spécialité FLE. Grenoble : Université Stendhal.

Gapaillard, C. \& Feutry Le Pennec, S. (2006). Maîtriser le discours scientifique. Lettres. Sciences de la vie et de la terre. Caen : CRDP.

Lüger, H.-H. (1993). « Routines conversationnelles et comportement langagier ». Langage et Société, $n^{\circ} 63$. pp. 7-38.

Mangiante, J.-M. \& Parpette, C. (2004). Le Français sur Objectif Spécifique : de l'analyse des besoins à l'élaboration d'un cours. Paris : Hachette.

Mangiante, J.-M. \& Parpette, C. (2011). Le Français sur Objectif Universitaire. Grenoble : PUG. Omer, D. (2003). « La prise de notes à la française pour des noteurs non natifs ». Arob[at]se, vol. 1-2. pp. 141-151. Disponible en ligne. https://halshs.archives-ouvertes.fr/hal-00416171/ document.

Parpette, C. \& Bouchard, R. (2003). « Gestion lexicale et prise de notes dans les cours magistraux ». Arob[at]se, vol. 1-2.pp 69-78. Disponible en ligne. https://hal.archives-ouvertes.fr/ hal-00376574.

Parpette, C. (2007). « Les cours magistraux : où situer les difficultés de compréhension ?». In Goes, J.\& Mangiante, J.-M. (dir.). L'accueil des étudiants étrangers dans les universités francophones. Arras : Artois Presses universités. pp. 49-60.

Samper Mangin, V. (2011). Comment adapter une formation en français à un public hétérogène d'étudiants internationaux. Conception d'un dispositif de Français sur Objectifs Universitaires à l'Institut Polytechnique de Grenoble. Mémoire de Sciences du Langage / spécialité FLE. Grenoble : Université Stendhal. http://dumas.ccsd.cnrs.fr/dumas-00578764/document

Toshimitsu Fujita, G. A. (2011). L'interculturalité et le FOS universitaire. Une étude pour la meilleure intégration des étudiants brésiliens dans les filières universitaires françaises. Mémoire de Sciences du Langage / spécialité FLE. Grenoble : Université Stendhal. http:// dumas.ccsd.cnrs.fr/dumas-00608308/document

Traverso, V. (1996). La conversation familière. Analyse pragmatique des interactions. Lyon : PUL.

\section{NOTES}

1. En moyenne, le taux de réussite des étudiants étrangers est $40 \%$ inférieur à celui des étudiants français (Mangiante \& Parpette, $2011: 17$ ).

2. Les sciences de l'ingénieur offrent un éventail très large de spécialités, et il nous importait donc de faire apparaitre, dans le matériel collecté, des aspects transversaux, communs aux différentes disciplines.

3. Dans les exemples cités tirés du corpus, la lettre $\mathrm{P}$ se réfère aux paroles de l'enseignant / professeur, la lettre E aux paroles des étudiants.

4. Le ton de l'enseignant est ici explicite, la fin de la phrase est suspendue.

5. Même s'il s'agit essentiellement d'un questionnement rhétorique, procédé didactique largement employé. 


\section{RÉSUMÉS}

Cette recherche s'inscrit dans le cadre du Français sur Objectif Universitaire, dans la perspective de la préparation linguistique des étudiants allophones souhaitant intégrer des filières universitaires scientifiques. Si dans un cours magistral, le format interactionnel exige rituellement que seul l'enseignant prenne la parole, il en va différemment lors des Travaux Dirigés (TD). Nous avons donc voulu observer les caractéristiques interactionnelles de ce type de cours, très présent dans les filières scientifiques, et qui a fait l'objet de moins de recherches que le cours magistral.Le corpus est constitué d'enregistrements vidéo de TD réalisés en école d'ingénieur. Nous avons observé et analysé les interactions enseignant / apprenants, en particulier lors de la correction collective des exercices. Cette analyse a fait apparaitre deux types de rituels langagiers sur lesquels nous nous attarderons : la co-construction de la solution et la négociation. Nous verrons que ces rituels et leur réalisation langagière constituent des indices du contrat didactique tacite entre enseignant et étudiants, indices qui peuvent être difficiles à interpréter par les étudiants allophones, en fonction de leur culture éducative et de leur niveau en français.

This research is cast in the French for Academic Purposes framework and sets out to examine the linguistic training of allophones students wishing to enroll in post-secondary scientific curriculums. During formal lectures, the interactional pattern typically requires that the teacher be the only one speaking. This is however not the case for tutorials. Our aim was therefore to observe the interactional characteristics of this type of practical lectures which have become a staplein scientific curriculums but have received less scholarly attention than traditional lectures. Our corpus consists of video recordings shot during tutorials in an Engineering School. We have observed and analyzed the interactions between teachers and learners, particularly during group corrections. Our analysis reveals two types of language rituals which we then proceed to discuss extensively: negociation, and the co-construction of solution. As we shall see, these rituals and their linguistic actualization are indicators of the implicit didactic agreement between teachers and students that can be hard to interpret by allophone students depending on their educational culture and their level in French.

\section{INDEX}

Mots-clés : français sur objectif universitaire, français scientifique, interactions didactiques, routines langagières

Keywords : french for academic purposes, french for scientific writing, classroom interactions, language habits 


\section{AUTEUR}

\section{CATHERINE CARRAS}

Université Grenoble Alpes, Bâtiment Stendhal UFR LLASIC- CS40700 - 38058 Grenoble cedex 9, France.

Catherine Carras est Maître de Conférences en Sciences du Langage à l'Université Grenoble Alpes, membre du laboratoire LIDILEM. Spécialiste de Français sur Objectif Spécifique, ses travaux portent majoritairement sur la préparation linguistique et méthodologique des étudiants non francophones devant intégrer des cursus universitaires francophones.

Courriel : Catherine.Carras[at]u-grenoble3.fr 